\title{
Prostatic Adenocarcinoma Metastatic to Pleomorphic Liposarcoma, a "Collision Phenomenon": Report of a Case with Review of Pelvic Collision Tumors
}

\author{
Somak Roy, ${ }^{1}$ Ronald L. Hrebinko, ${ }^{2}$ Kathleen M. Cieply, ${ }^{3}$ \\ Anil V. Parwani, ${ }^{4}$ and Uma N. M. Rao ${ }^{5}$ \\ ${ }^{1}$ Department of Pathology, University of Pittsburgh Medical Center, A615, Scaife Hall, 3550 Terrace Street, Pittsburgh, \\ PA 15261, USA \\ ${ }^{2}$ Department of Urology, University of Pittsburgh Medical Center, Suite 209, Shadyside Medical Building, 5200 Centre Avenue, \\ Pittsburgh, PA 15232, USA \\ ${ }^{3}$ Department of Pathology, University of Pittsburgh Medical Center (UPMC), 631E Montefiore Hospital, Pittsburgh, \\ PA 15261, USA \\ ${ }^{4}$ Department of Pathology, UPMC Shadyside Hospital, Room Wing 02.10, 5230 Centre Avenue, Pittsburgh, PA 15232, USA \\ ${ }^{5}$ Department of Pathology, University of Pittsburgh Medical Center (UPMC), Room 2.9 West Wing, Shadyside, Pittsburhg, \\ PA 15232, USA
}

Correspondence should be addressed to Somak Roy, roys911@gmail.com

Received 6 March 2011; Accepted 17 June 2011

Academic Editor: R. Montironi

Copyright (c) 2011 Somak Roy et al. This is an open access article distributed under the Creative Commons Attribution License, which permits unrestricted use, distribution, and reproduction in any medium, provided the original work is properly cited.

\begin{abstract}
"Collision tumor" is an uncommon phenomenon characterized by coexistence of two completely distinct and independent tumors at the same site. Collision tumors have been reported in different sites in the body; however, these are particularly uncommon in the pelvic cavity. A 70-year-old man, with prior history of urothelial and prostate cancer, presented with a large pelvic mass detected on imaging studies. Pathological examination revealed a large liposarcoma with prostatic carcinoma embedded in it. Immunohistochemistry and florescence in situ hybridization studies were performed to reach to a conclusive diagnosis. To the best of our knowledge, this is the second case reported till date. We present the challenges encountered in the diagnosis of this case and review of pelvic collision tumors.
\end{abstract}

\section{Introduction}

"Collision tumor" is a well-documented but uncommon phenomenon characterized by coexistence of two completely distinct and independent tumors at the same site [1-11]. The two morphologically separate tumors, which are genetically different, are sharply demarcated from each other. This entity is distinct from neoplasms demonstrating heterologous elements, as a result of divergent differentiation $[1,4]$.

The previously documented cases of collision tumor are predominantly synchronous epithelial tumors with different lineages such as melanoma occurring in a basal cell carcinoma [10] or gastric adenocarcinoma within a gastrointestinal stromal tumor [11], tumor to tumor metastasis [1, 2, 4, 12,13 , tumors coexisting as part of a systemic process (small cell lymphoma/chronic lymphocytic leukemia with pelvic myelolipoma and renal cell carcinoma with intravascular lymphomatosis) $[5,6]$, and two coexistent metastatic tumors in a single lymph node [14-21]. The last category is rare and has also been referred to as "collision metastasis" $[14,16]$. The various collision tumors have been documented in different regions of the body including head and neck, thorax, abdomen, and pelvic cavity. The incidence of carcinoma metastasizing to mesenchymal tumor, especially in the pelvis, is extremely rare $[3,7,9,12,22]$. We present a case of a patient with both urothelial and prostate cancers where in the prostatic carcinoma metastasized to a retroperitoneal pleomorphic liposarcoma. The lineages of both tumors were confirmed by immunohistochemistry and florescence in situ hybridization (FISH). 


\section{Clinical History}

The patient was a 70-year-old man with a surgical history significant for high-grade urothelial carcinoma of urinary bladder. In 2003 he presented with painless hematuria and was found to have a tumor confined entirely inside a large urinary bladder diverticulum. Cystoscopy confirmed no tumor in the bladder cavity, and a pelvic CT did not demonstrate any evidence of extravesical tumor spread or metastatic disease. Microscopic examination of the prior bladder tumor demonstrated mixture of highly atypical malignant epithelioid and spindle cells (Figure 1), which demonstrated diffuse immunoexpression of pancytokeratin and cytokeratin 20. The tumor cells were negative for vimentin and CD68. The features were diagnostic of highgrade sarcomatoid urothelial carcinoma. He underwent partial cystectomy followed by adjuvant chemotherapy and intravesical BCG. In 2006, he had radical retropubic prostatectomy and radiotherapy for a prostate adenocarcinoma, Gleason's score $4+4=8$ (Figure 2). Extracapsular extension, seminal vesicle, and perineural and angiolymphatic invasion were found on pathological examination. There were no palpable lymph nodes; computed tomography did not show any evidence of lymph node metastasis, and there was no recurrence of urothelial carcinoma.

Forty-five months later, a steady rise in serum prostatespecific antigen (PSA) from $1.9 \mathrm{ng} / \mathrm{mL}$ to $8.9 \mathrm{ng} / \mathrm{mL}$ was noted over the last 4 months. A surveillance cystoscopy showed evidence of extrinsic bladder compression which on digital rectal examination was felt to be a firm mass. Computed tomography with contrast of the pelvis and abdomen showed a large, $18 \times 12.7 \mathrm{~cm}$ heterogeneous solidcystic mass with irregular internal enhancement. The mass, extended from the pelvis up to the transverse segment of the duodenum, involved the dome of the bladder, left pelvic side wall, left ureterovesical junction, and rectosigmoid colon. There was evidence of left-sided obstructive hydroureter and hydronephrosis due to the tumor. No evidence of metastasis was seen in thorax and upper abdomen.

The patient underwent resection of the tumor with en bloc sigmoid colectomy and ureteroneocystostomy. Intraoperatively the large mass was visualized which appeared to be arising from pelvis with definite invasion into the sigmoid colon, involving lower portion of left ureter and portion of the dome of bladder. A small irregular lesion was noted in the lower retrovesicle region which was found to be welldifferentiated prostatic adenocarcinoma on intraoperative frozen section. The mass was entirely resected and sent for pathological examination.

\section{Pathologic Findings}

3.1. Gross Findings. The resected specimen was a large, well circumscribed, nonencapsulated, and yellow-pink multinodular $18.5 \times 16.0 \times 11.5 \mathrm{~cm}$ mass with an attached segment $32.5 \mathrm{~cm}$ of large intestine (Figure $3(\mathrm{a})$ ). The cut surfaces of the mass were firm with multiloculated cysts, focally hemorrhagic, and variegated tan-brown to red. The cystic

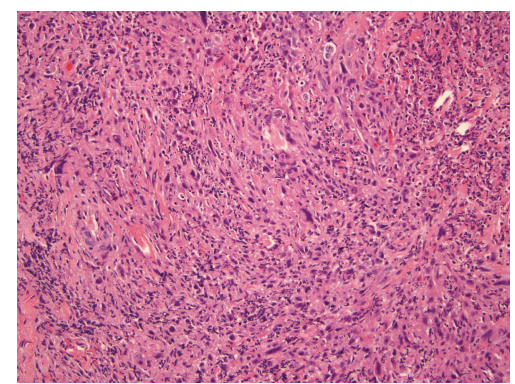

FIGURE 1: Sarcomatoid urothelial carcinoma. Tumor comprised of sheets of epithelioid and plump spindle cells which are highly pleomorphic with nuclear hyperchromasia. There is prominent stromal inflammatory response to the invading tumor with destruction of the bladder wall. (Hematoxylin \& eosin, original magnification $\times 100)$.

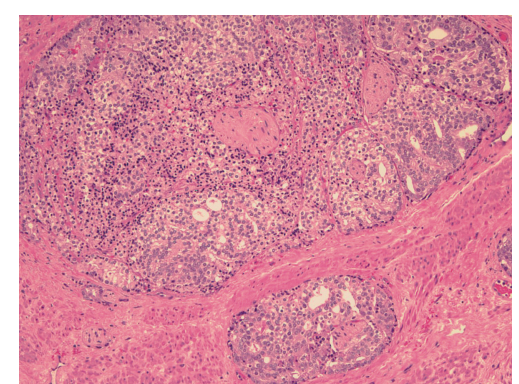

FIgure 2: Prostatic adenocarcinoma: The large nodules of tumor cells, invading the stroma, are arranged mainly as sheets of cells and fused acini. Prominent perineural invasion is identified. (Hematoxylin \& eosin, original magnification $\times 100$ ).

structures were smooth-walled, filled with serosanguinous fluid, and some had ragged and nodular tan-yellow lining.

3.2. Microscopic Findings. The mass was extensively sampled, and subsequent histologic examination demonstrated a malignant mesenchymal tumor with variably cellular stroma. The cellular areas were comprised of large cells with moderate to abundant clear to eosinophilic cytoplasm, enlarged, highly pleomorphic, and vesicular nuclei with distinct nucleolus, admixed with scattered pleomorphic and bizarre multinucleated giant tumor cells (Figure 3(b)). Scattered lipoblasts were noted in the cellular areas with prominent plexiform vasculature (Figures $3(\mathrm{c})$ and $3(\mathrm{~d})$ ). The stroma in the hypocellular areas was sclerotic to focally myxoid with few scattered spindle cells. Also seen dispersed in the hypocellular areas were collection of glandular tumor, morphologically similar to the prostate carcinoma, lined by cuboidal to columnar cells with eosinophilic to clear cytoplasm and enlarged vesicular nuclei with prominent nucleoli (Figures 3(e), 3(f)). Tumor was also noted in the retrovesical tissue.

Immunohistochemical analysis revealed immunoexpression of cytokeratin AE1/AE3 (Monoclonal Mouse, DAKO, 1:100) (Figure 4(a)), CAM5.2 (Monoclonal Mouse, BD, $1: 10$ ), focal and weak P501S (Monoclonal Mouse, DAKO, 


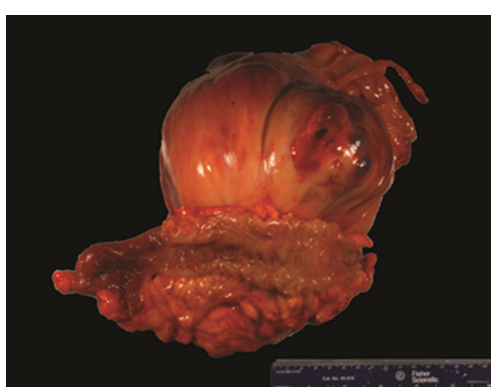

(a)

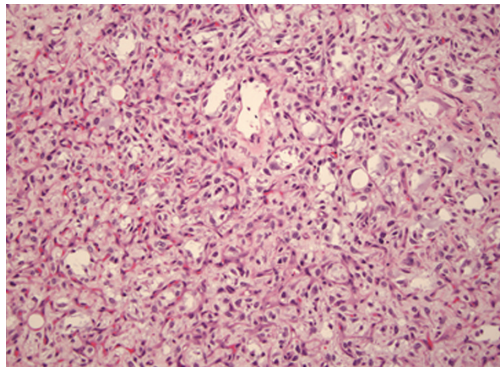

(d)

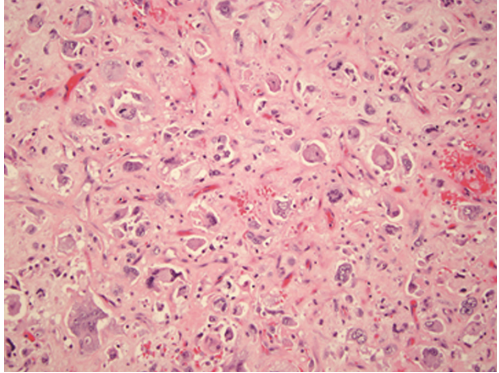

(b)

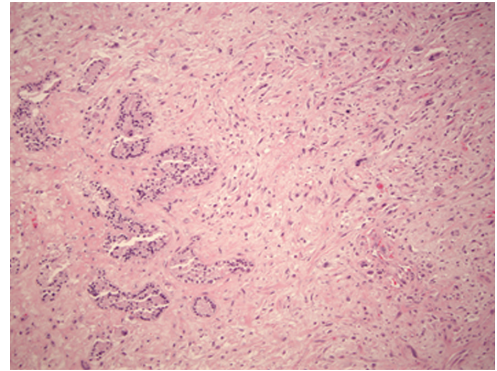

(e)

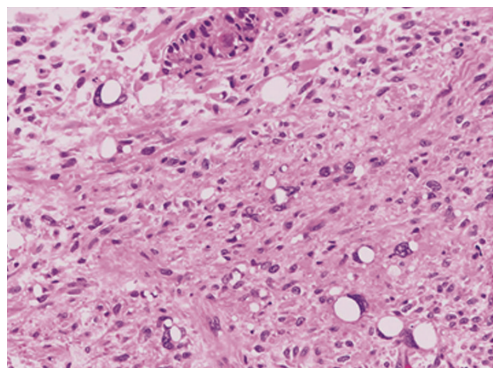

(c)

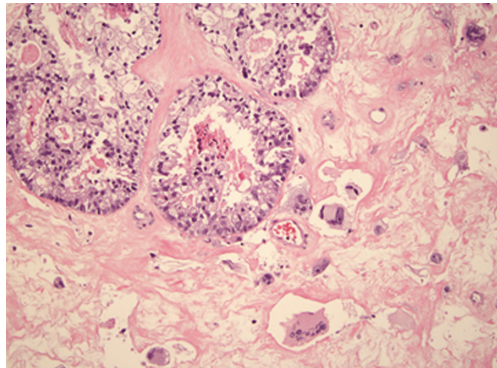

(f)

Figure 3: Image Plate I. (a) Gross image of the pelvic mass resection. The mass was large, well circumscribed, nonencapsulated and yellowpink with an attached segment of large intestine. (b) Cellular area of the tumor, comprised of large cells with enlarged, highly pleomorphic, and vesicular nuclei with distinct nucleoli and abundant, clear to eosinophilic cytoplasm, with scattered bizarre multinucleated tumor giant cells. The stroma appears hyalinized with delicate branching capillary network. (Hematoxylin \& eosin, original magnification $\times 200$.) (c) Scattered lipoblasts in a variably cellular background. (Hematoxylin \& eosin, original magnification $\times 400$.) (d) Highly cellular area of tumor showing plexiform network of delicate capillaries, admixed tumor cells, and rare lipoblast. (Hematoxylin \& eosin, original magnification $\times 200$.) (e) Glandular tumor aggregate comprised of variably sized acini (left), scattered in a hyalinized stroma. Interface with the liposarcoma component (right) is clearly seen. (Hematoxylin \& eosin, original magnification $\times 40$.) (f) Close-up view of the prostatic carcinoma showing well formed acini and inspissated eosinophilic luminal secretion. The acini are intricately admixed with the bizarre tumor giant cells of the liposarcoma in a myxoid to hyalinized stroma.

$1: 40$ ), and androgen receptor (AR) (Monoclonal Mouse, DAKO, 1:100) in the glandular component. The myxoid and pleomorphic component was strongly positive for vimentin (Monoclonal Mouse, Ventana, Prediluted) (Figure 4(b)) and negative for several cytokeratin markers, S-100 (Polyclonal rabbit, DAKO, 1:500), HMB45 (Monoclonal Mouse, Ventana, Prediluted), Melan-A (Monoclonal Mouse, DAKO, 1:100), demsin (Monoclonal Mouse, Ventana, Prediluted), smooth muscle actin (SMA) (Monoclonal Mouse, Cell Marque, Prediluted), myogenin (Monoclonal Mouse, Cell Marque, Prediluted), and CD117 (Polyclonal rabbit, DAKO, 1 : 100). CD34 (Monoclonal Mouse, Ventana, Prediluted) highlighted the prominent plexiform vasculature (Figure 4(c)).

Florescence in situ hybridization (FISH) analysis, performed using dual-color break-apart probe for $\mathrm{CHOP}$ (CHOP dual-color break-apart probe, Abbott Molecular, Des Plaines, Ill) gene (Figure 4(d)), was positive for the translocation pattern and negative for MDM2 amplification (Figure 4(e)) (MDM2 clones RP11-450G15, RP11-775J10 (CHORI)/CEP12 Abbott Molecular, Des Plaines, Ill). FISH analysis using dual-color double-fusion probes for TMPRSS2 (clone RP11-24A11 (CHORI, Oakland, Calif)) and ERG (clone RP11-35C4 (CHORI), Oakland, Calif), demonstrated the fusion transcript in the focus harboring prostatic adenocarcinoma (Figure 4(f)).
The patient is alive at 7 months followup, but has a rising PSA from 6.1 to $8.6 \mathrm{ng} / \mathrm{mL}$. Magnetic resonance imaging revealed a $4.2 \mathrm{~cm}$ left pelvic cavity mass concerning for recurrent/residual tumor.

\section{Discussion}

Collision tumors represent an uncommon coexistence of tumors with distinct morphology and biology. These tumors occurring in the pelvic cavity are rare with only 8 cases being reported till date $[2-5,7,9,12,22]$ (Table 1 ) of which prostate adenocarcinoma was the most frequent component tumor. There are 4 reported cases with soft-tissue tumor as one of the components (two leiomyosarcoma [3, 9], one leiomyoma [22], and one liposarcoma [7]). Only 3 cases of collision tumors with liposarcoma as one of the components have been reported $[1,7,24]$. The collision tumor of prostate adenocarcinoma and liposarcoma has been documented previously as a single case report in 1978 by Juhasz and Kiss, the current case being the second one reported till date.

In our current case, the patient had past history of a urothelial and a prostate carcinoma, followed by the liposarcoma (Table 2). Each epithelial tumor was treated surgically followed by chemotherapy and radiation. There was an approximate three-year interval between occurrence 


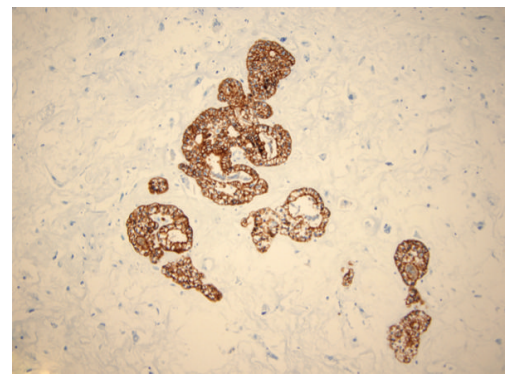

(a)

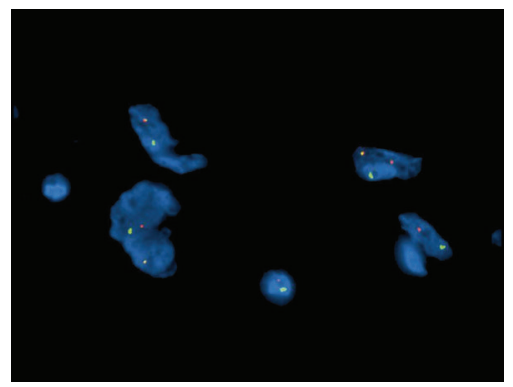

(d)

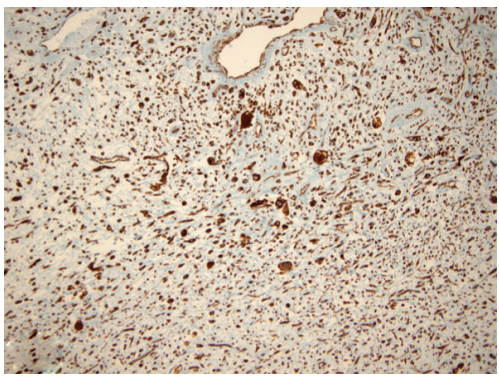

(b)

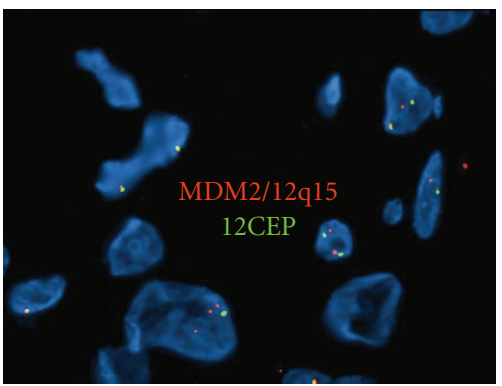

(e)

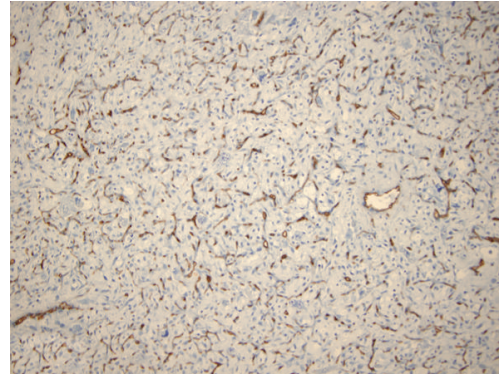

(c)

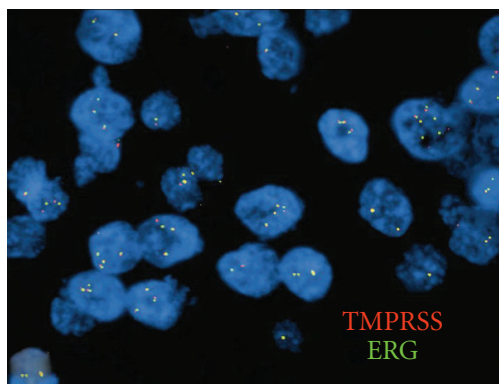

(f)

FIGURE 4: Image Plate II. (a) Malignant prostatic acini are strongly positive for AE1/AE3. The spindle cells are negative. (Original magnification $\times 100$.) (b) Strong expression of vimentin in the liposarcoma component. (Original magnification $\times 40$.) $(\mathrm{c})$ CD34 highlights the plexiform network of capillaries in the tumor. (Original magnification $\times 40$.) (d) FISH analysis, dual-color break-apart probe for CHOP is positive for the translocation, represented by a cell with one yellow signal (normal), one red (part of CHOP gene), and one green signal (part of CHOP gene). (e) FISH analysis, amplification probe for MDM2, is negative (not amplified). (f) FISH analysis, dual-color fusion probes for TMPRSS (red) and ERG (green), is positive, represented by cells with at least one fused yellow signal (red + green).

TABLE 1: List of collision tumors in the pelvis.

\begin{tabular}{lcccc}
\hline Case no. & Source & Tumor 1 & Tumor 2 & Site \\
\hline$(1)$ & Juhasz and Kiss/1978 [7] & Liposarcoma & Pr ACa & Right seminal vesicle \\
$(2)$ & Erler/1978 [22] & Pleomorphic carcinoma & Leiomyoma & True pelvis \\
$(3)$ & Palma et al./1983 [9] & Leiomyosarcoma & Pr ACa & Prostate \\
$(4)$ & Oda et al./1984 [23] & TCC bladder & Colon ACa & Vesicocolic fistula \\
$(5)$ & Paveli et al./2000 [3] & Rectal leiomyosarcoma & Pr ACa & Prostate \& rectum \\
$(6)$ & Roh et al./2006 [2] & Rectal ACa & Rectum \\
$(7)$ & Brahmania et al./2007 [4] & Colon ACa & Granulosa cell tumor, ovary & Pelvic mass \\
$(8)$ & Gheith et al./2009 [5] & Myelolipoma & CLL/SLL & Pelvic mass \\
\hline
\end{tabular}

PrACa: prostatic adenocarcinoma, ACa: adenocarcinoma, TCC: transitional cell carcinoma, CLL/SLL: chronic lymphocytic leukemia/small lymphocytic lymphoma.

of the tumors, each of which was extensively studied, and diagnosis confirmed by immunohistochemistry.

It is not certain whether the liposarcoma had been present during the initial or subsequent surgeries, since retroperitoneal sarcomas, especially liposarcomas, can manifest when they attain large size, but in this patient, each epithelial malignancy was staged appropriately, and at diagnosis, no other intra-abdominal masses were detected. It is possible that the liposarcoma was smaller and differentiated at inception and had a growth spurt when dedifferentiation occurred.

Pathological examination revealed a liposarcoma with embedded prostatic adenocarcinoma. The diagnosis in this case was challenging due to paucity of characteristic lipoblasts in the high-grade sarcoma and some morphologic similarity with the patient's prior bladder tumor, thus necessitating the use of ancillary tests, especially FISH, for the liposarcoma-related gene targets.

Collision metastasis to pelvic lymph nodes is a rare event and most frequently involved by prostate adenocarcinoma in combination with colonic adenocarcinoma and bladder carcinoma [15-20]. In this case, we describe that prostatic adenocarcinoma was scattered throughout the liposarcoma, which suggests that this best represents a tumor (prostate adenocarcinoma) to tumor (liposarcoma) metastasis, since no residual lymph node architecture was found. 
TABLE 2: Sequence of events.

\begin{tabular}{|c|c|c|c|c|c|c|}
\hline Year & Site & Morphology & IHC & FISH & Diagnosis & Treatment \\
\hline $5 / 2003$ & $\begin{array}{l}\text { Urinary } \\
\text { bladder } \\
\text { diverticulum }\end{array}$ & $\begin{array}{l}\text { Biphasic (epithelial and } \\
\text { spindle cells) tumor with } \\
\text { high-grade cytologic } \\
\text { features. ALI-, PNI-, } \\
\text { perivesical soft-tissue } \\
\text { invasion }\end{array}$ & $\begin{array}{l}\text { panCK+, } \\
\text { CK20+, } \\
\text { Vimentin-, } \\
\text { CD68-, } \\
\text { desmin-, } \\
\text { SMA- }\end{array}$ & NA & $\begin{array}{l}\text { Sarcomatoid } \\
\text { urothelial } \\
\text { carcinoma }\end{array}$ & $\begin{array}{l}\text { Partial cystectomy, } \\
\text { gemcitabine and } \\
\text { carboplatin ( } 4 \text { cycles), } \\
\text { intravesical BCG }\end{array}$ \\
\hline $10 / 2006$ & Prostate & $\begin{array}{l}\text { Prostate adenocarcinoma, } \\
\text { Gleason's Score } 4+4=8 \text {, } \\
\text { ALI+, PNI+, ECE }+, \text { Seminal } \\
\text { vesicle involvement. }\end{array}$ & NA & NA & $\begin{array}{l}\text { High-grade } \\
\text { prostatic } \\
\text { adenocarcinoma }\end{array}$ & $\begin{array}{l}\text { Radical prostatectomy } \\
\text { and radiotherapy }\end{array}$ \\
\hline $4 / 2010$ & Pelvic mass & $\begin{array}{l}\text { Myxoid liposarcoma with } \\
\text { pleomorphic areas, } \\
\text { characteristic lipoblasts and } \\
\text { hyalinized stroma mingled } \\
\text { with well-differentiated } \\
\text { prostatic adenocarcinoma }\end{array}$ & $\begin{array}{l}\text { Liposarcoma: } \\
\text { vimentin+, } \\
\text { S100-, SMA-, } \\
\text { desmin-, } \\
\text { HMB45-, } \\
\text { melan A-, } \\
\text { myogenin-, } \\
\text { CD117- } \\
\text { PCa: AE1/AE3+, } \\
\text { CAM5.2+, weak } \\
\text { P501S+, AR+ }\end{array}$ & $\begin{array}{l}\text { Liposarcoma: } \\
\text { CHOP+, } \\
\text { MDM2- } \\
\text { PCa:TMPRSS2- } \\
\text { ERG+ }\end{array}$ & $\begin{array}{l}\text { Dedifferentiated } \\
\text { myxoid } \\
\text { liposarcoma } \\
\text { with embedded } \\
\text { prostatic } \\
\text { adenocarcinoma }\end{array}$ & $\begin{array}{l}\text { Tumor resection, en } \\
\text { bloc sigmoid } \\
\text { colectomy, and } \\
\text { ureteroneocystostomy }\end{array}$ \\
\hline
\end{tabular}

ALI: angiolymphatic invasion, AR: androgen receptor, CK: Cytokeratin, ECE: extracapsular extension, FISH: florescence in situ hybridization, IHC: immunohistochemistry, PCa: prostatic adenocarcinoma: PNI: perineural invasion, SMA: smooth muscle actin.

Use of immunohistochemical markers helped in establishing the diagnosis of prostate adenocarcinoma based on immunoreactivity for pancytokeratin, CAM5.2, P501S, and AR. The sarcomatous component expressed vimentin only and was negative for an entire battery of immunostains.

Determination of loss of heterozygosity ( $\mathrm{LOH}$ ) and polymerase chain reaction (PCR) can be used to help differentiate the components in a tumor to tumor metastasis $[2,3,5]$. We used dual-color FISH to confirm diagnosis of liposarcoma, because of the presence of pleomorphic areas rather than the round cell component generally associated with highgrade myxoid liposarcoma. CHOP gene, mapped to the long arm of chromosome (12q23) is also known as DDIT3, encodes a member of the CCAAT/enhancer-binding protein (C/EBP) family of transcription factors which is implicated in adipogenesis and erythropoiesis. The FUS gene, present on chromosome 16, encodes for an RNA-binding protein [25, 26]. Chromosomal translocation $t(12 ; 16)$ (q13;p11) leads to fusion of $5^{\prime}$ end of FUS gene (exons $1-5$, promoter region) and complete coding region of $C H O P$ which causes stable expression of the fusion protein leading to tumor formation $[26,27]$. This translocation is characteristic of myxoid liposarcomas and seen in 95\% of the cases. Three types of fusion transcripts have been reported based on the length of the promoter portion of FUS gene, type II being the most common [26]. The remainder 5\% of myxoid liposarcomas have another translocation, $t(12 ; 22)(\mathrm{q} 13 ; \mathrm{q} 12)$, involving CHOP and EWSR1 genes [26]. FISH analysis using CHOP dual-color, break-apart probe has been reported to be the most efficient and sensitive method for diagnosis of myxoid liposarcomas [25]. In our case, the above FISH analysis helped in the diagnosis of dedifferentiated liposarcoma and also ruled out the possibility of metastasis from prior bladder sarcomatoid carcinoma.

MDM2 gene region amplification, characterized by the presence of giant marker/ring chromosome 12 containing MDM2 sequences, is seen in atypical lipomatous tumor/welldifferentiated liposarcoma and dedifferentiated liposarcomas [28]. However, the specificity of the MDM2 FISH analysis is lower in the later case, as other pleomorphic sarcomas may also show MDM2 amplification $[25,26]$. In the current case FISH for MDM2 analysis was negative.

$E R G$, a member of the ETG family of transcription factors, functions in conjunction with other transcription factors in regulation of many cellular functions, proliferation, differentiation, oncogenesis, and angiogenesis [29]. TMPRSS2 is predominantly expressed in the luminal epithelial cells of prostatic acini. Both the genes are located $3 \mathrm{Mb}$ apart on the long arm of chromosome 21 (q22.2-22.3). Interstitial deletion of the intervening intronic DNA in this region is the most common mechanism for formation of the TMPRSS2-ERG fusion transcript, which is seen in about half of prostate cancers [29-31]. This rearrangement can be detected either using FISH or reverse transcriptase PCR (RTPCR). We performed FISH analysis using dual-fusion probes for TMPRSS2 and ERG, which demonstrated the fusion transcript in the foci of prostatic adenocarcinoma. Although prostate carcinomas associated with this gene rearrangement have been reported to have a poor clinical outcome and decreased overall survival, the data available till date is still controversial [29].

The underlying pathobiology of collision tumors is still uncertain; however, various hypotheses have been postulated [1] as follows. 
(1) Occurrence of two different tumor types in one site is coincidental.

(2) A single carcinogenic stimulus alters the environment of the region conducive for occurrence of two different tumors.

(3) Presence of first tumors alters the microenvironment favorable for development of the second tumor.

In this multiple cancer-prone individual with no history of familial cancers, several intriguing possibilities exist. (a) Most of the high-grade retroperitoneal sarcomas are dedifferentiated liposarcomas that are frequently de novo or have dedifferentiated from a preexisting low-grade liposarcoma; the latter is most likely a temporal event $[26,32,33]$. Liposarcomas for practical purposes do not arise from preexisting lipomas [26]. Radiation-induced dedifferentiation in liposarcomas is unlikely and has not been reported for prostate carcinomas also. (b) All three neoplasms in the current case must have been synchronous tumors that became clinically manifested at different time frames. (c) In majority of tumor to tumor metastases, the recipient is usually a richly vascular tumor as argued in previous reports $[34,35]$. Myxoid liposarcoma are also richly vascular and contain distinct plexiform vasculature and, therefore, can be considered as a recipient of the prostate adenocarcinoma since no residual lymph node architecture was found.

In conclusion we have documented a rare case of prostate adenocarcinoma that most likely metastasized to a liposarcoma and grew undetected in a large dedifferentiated or pleomorphic myxoid liposarcoma. It is also of interest that this CHOP FUS-positive poorly differentiated myxoid liposarcoma did not contain the round cell component associated with myxoid liposarcoma, but this has been described to occur in some myxoid liposarcomas [36].

The use of morphological features, immunohistochemistry, and appropriate cytogenetic tests helped in reaching a correct diagnosis. The emphasis is on awareness amongst clinician, and pathologists of existence of such a collision tumor and role of extensive tumor sampling and appropriate use of ancillary tests to arrive at a diagnosis.

\section{References}

[1] M. Brandwein-Gensler, M. Urken, and B. Wang, "Collision tumor of the thyroid: a case report of metastatic liposarcoma plus papillary thyroid carcinoma," Head and Neck, vol. 26, no. 7, pp. 637-641, 2004.

[2] Y. H. Roh, H. W. Lee, M. C. Kim, K. W. Lee, and M. S. Roh, "Collision tumor of the rectum: a case report of metastatic gastric adenocarcinoma plus primary rectal adenocarcinoma," World Journal of Gastroenterology, vol. 12, no. 34, pp. 55695572, 2006.

[3] J. Paveli, J. Lamovec, J. Novak, K. Gall-Troselj, S. Kapitanovic, and K. Pavelic, "Collision tumor in the pelvic cavity: rectal leiomyosarcoma and prostate adenocarcinoma," Journal of Cancer Research and Clinical Oncology, vol. 126, no. 2, pp. 95100, 2000.

[4] M. Brahmania, C. S. Kanthan, and R. Kanthan, "Collision tumor of the colon-colonic adenocarcinoma and ovarian granulosa cell tumor," World Journal of Surgical Oncology, vol. 5, article 118, 2007.

[5] S. Gheith, R. Boulay, and D. Cornfield, "Small lymphocytic lymphoma/chronic lymphocytic leukemia in a pelvic myelolipoma," International Journal of Clinical and Experimental Pathology, vol. 2, no. 1, pp. 95-98, 2009.

[6] B. Y. Wang, J. A. Strauchen, D. Rabinowitz, S. M. Tillem, and P. D. Unger, "Renal cell carcinoma with intravascular lymphomatosis: a case report of unusual collision tumors with review of the literature," Archives of Pathology and Laboratory Medicine, vol. 125, no. 9, pp. 1239-1241, 2001.

[7] J. Juhasz and P. Kiss, "A hitherto undescribed case of "collision" tumour: liposarcoma of the seminal vesicle and prostatic carcinoma," International Urology and Nephrology, vol. 10, no. 3, pp. 185-193, 1978.

[8] A. P. Hart, R. Brown, J. Lechago, and L. D. Truong, "Collision of transitional cell carcinoma and renal cell carcinoma: an immunohistochemical study and review of the literature," Cancer, vol. 73, no. 1, pp. 154-159, 1994.

[9] P. C. Palma, N. Rodrigues Neto Junior, O. Ikari, C. A. D'Ancona, and A. Billis, "Leiomyosarcoma in association with incidental adenocarcinoma of the prostate," Journal of Urology, vol. 129, no. 1, pp. 156-157, 1983.

[10] J. Rodriguez, D. Nonaka, E. Kuhn, M. Reichel, and J. Rosai, "Combined high-grade basal cell carcinoma and malignant melanoma of the skin ("malignant basomelanocytic tumor"): report of two coses and review of the literature," American Journal of Dermatopathology, vol. 27, no. 4, pp. 314-318, 2005.

[11] B. Kleist, J. Lasota, and M. Miettinen, "Gastrointestinal stromal tumor and gastric adenocarcinoma collision tumors," Human Pathology, vol. 41, no. 7, pp. 1034-1039, 2010.

[12] L. Doering, "Metastasis of carcinoma of prostate to meningioma. Case report," Virchows Archiv, vol. 366, no. 1, pp. 8791, 1975.

[13] A. Fornelli, A. Bacci, G. Collina, and V. Eusebi, "Breast carcinoma metastatic to meningioma: review of the literature and description of 2 new cases," Pathologica, vol. 87, no. 5, pp. 506-512, 1995.

[14] M. A. Sughayer, L. Zakarneh, and R. Abu-Shakra, "Collision metastasis of breast and ovarian adenocarcinoma in axillary lymph nodes: a case report and review of the literature," Pathology and Oncology Research, vol. 15, no. 3, pp. 423-427, 2009.

[15] A. D. Morgen, "Carcinomas of rectum and prostate metastasizing to the same lymph nodes," The Journal of Pathology, vol. 97, no. 1, pp. 143-145, 1969.

[16] Z. K. Wade, J. E. Shippey, G. A. Hamon, R. L. Smoak, P. Argani, and W. C. Allsbrook, "Collision metastasis of prostatic and colonic adenocarcinoma: report of 2 cases," Archives of Pathology and Laboratory Medicine, vol. 128, no. 3, pp. 318320, 2004.

[17] N. Mourra, Y. Parc, D. McNamara, E. Tiret, J. F. Flejou, and R. Parc, "Lymph node metastases of prostatic adenocarcinoma in the mesorectum in patients with adenocarcinoma or villous tumor of the rectum with collision phenomenon in a single lymph node: report of five cases," Diseases of the Colon and Rectum, vol. 48, no. 2, pp. 384-389, 2005.

[18] A. Ergen, M. D. Balbay, M. Irwin, and R. Torno, "Collision metastasis of bladder and prostate carcinoma to a single pelvic lymph node," International Urology and Nephrology, vol. 27, no. 6, pp. 743-745, 1995.

[19] K. Gohji, M. Nomi, T. Kizaki, S. Maruyama, K. Morisue, and A. Fujii, 'Collision phenomenon' of prostate and bladder 
cancers in lymph node metastases," International Journal of Urology, vol. 4, no. 2, pp. 222-224, 1997.

[20] K. Overstreet and P. Haghighi, "Urothelial and prostate carcinoma metastasizing to the same lymph node: a case report and review of the literature," Archives of Pathology and Laboratory Medicine, vol. 125, no. 10, pp. 1354-1357, 2001.

[21] T. Terada, Y. Satoh, N. Aoki, R. Hirayama, Y. Ishikawa, and S. Hatakeyama, "The coexistence of cancer cells of different origin within the same lymph nodes," Surgical and Radiologic Anatomy, vol. 15, no. 2, pp. 119-123, 1993.

[22] G. Erler, "“Collision tumour", pleomorphic carcinoma and proliferative leiomyoma of the true pelvis. Anatomoclinical observation," Archives d'Anatomie et de Cytologie Pathologiques, vol. 26, no. 3-4, pp. 170-173, 1978.

[23] Y. Oda, G. Hamami, K. Umezu et al., "Vesicocolic fistula formed by "collision" tumor between transitional cell carcinoma of urinary bladder and adenocarcinoma of the sigmoid colon," Hinyokika Kiyo, vol. 30, no. 1, pp. 55-58, 1984.

[24] M. Orditura, E. Lieto, F. Ferraraccio et al., "Hepatoid carcinoma colliding with a liposarcoma of the left colon serosa presenting as an abdominal mass," World Journal of Surgical Oncology, vol. 5, article 42, 2007.

[25] M. R. Tanas and J. R. Goldblum, "Fluorescence in situ hybridization in the diagnosis of soft tissue neoplasms: a review," Advances in Anatomic Pathology, vol. 16, no. 6, pp. 383-391, 2009.

[26] S. W. Weiss and J. R. Goldblum, Enzinger and Weiss's Soft Tissue Tumors, Mosby, Philadelphia, Pa, USA, 5th edition, 2008.

[27] M. Sánchez-Martín, I. González-Herrero, and I. SánchezGarcía, "Soft Tissue Tumors: Liposarcoma: Myxoid liposarcoma," Atlas of Genetics and Cytogenetics in Oncology and Haematology, 2007, http://AtlasGeneticsOncology.org /Tumors/MyxoidLipoSarcID5169.html.

[28] A. Mariño-Enríquez, C. D. M. Fletcher, P. D. Cin, and J. L. Hornick, "Dedifferentiated liposarcoma with "homologous" lipoblastic (Pleomorphic Liposarcoma-like) differentiation: clinicopathologic and molecular analysis of a series suggesting revised diagnostic criteria," American Journal of Surgical Pathology, vol. 34, no. 8, pp. 1122-1131, 2010.

[29] A. C. Mackinnon, B. C. Yan, L. J. Joseph, and H. A. AlAhmadie, "Molecular biology underlying the clinical heterogeneity of prostate cancer: an update," Archives of Pathology and Laboratory Medicine, vol. 133, no. 7, pp. 1033-1040, 2009.

[30] J. Wang, Y. Cai, C. Ren, and M. Ittmann, "Expression of variant TMPRSS2/ERG fusion messenger RNAs is associated with aggressive prostate cancer," Cancer Research, vol. 66, no. 17, pp. 8347-8351, 2006.

[31] R. Mehra, S. A. Tomlins, R. Shen et al., "Comprehensive assessment of TMPRSS2 and ETS family gene aberrations in clinically localized prostate cancer," Modern Pathology, vol. 20, no. 5, pp. 538-544, 2007.

[32] E. Fabre-Guillevin, J. M. Coindre, N. De Saint Aubain Somerhausen, F. Bonichon, E. Stoeckle, and N. B. Bui, "Retroperitoneal liposarcomas: follow-up analysis of dedifferentiation after clinicopathologic reexamination of 86 liposarcomas and malignant fibrous histiocytomas," Cancer, vol. 106, no. 12, pp. 2725-2733, 2006.

[33] D. R. Lucas, A. G. Nascimento, B. K. S. Sanjay, and M. G. Rock, "Well-differentiated liposarcoma: the Mayo Clinic experience with 58 cases," American Journal of Clinical Pathology, vol. 102, no. 5, pp. 677-683, 1994.

[34] O. L. Bohn, L. E. de las Casas, and M. E. Leon, “Tumor-totumor metastasis: renal cell carcinoma metastatic to papillary carcinoma of thyroid-report of a case and review of the literature," Head and Neck Pathology, vol. 3, no. 4, pp. 327330, 2009.

[35] D. T. Cooke, D. J. Zheng, K. A. Peterson, and R. F. Calhoun, "Synchronous pulmonary renal cell carcinoma metastases and primary non-small cell lung cancer," Journal of Thoracic Oncology, vol. 5, no. 1, pp. 140-141, 2010.

[36] R. Alaggio, C. M. Coffin, S. W. Weiss et al., "Liposarcomas in young patients: a study of 82 cases occurring in patients younger than 22 years of age," American Journal of Surgical Pathology, vol. 33, no. 5, pp. 645-658, 2009. 


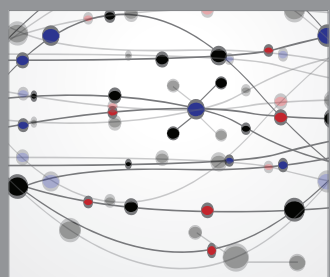

The Scientific World Journal
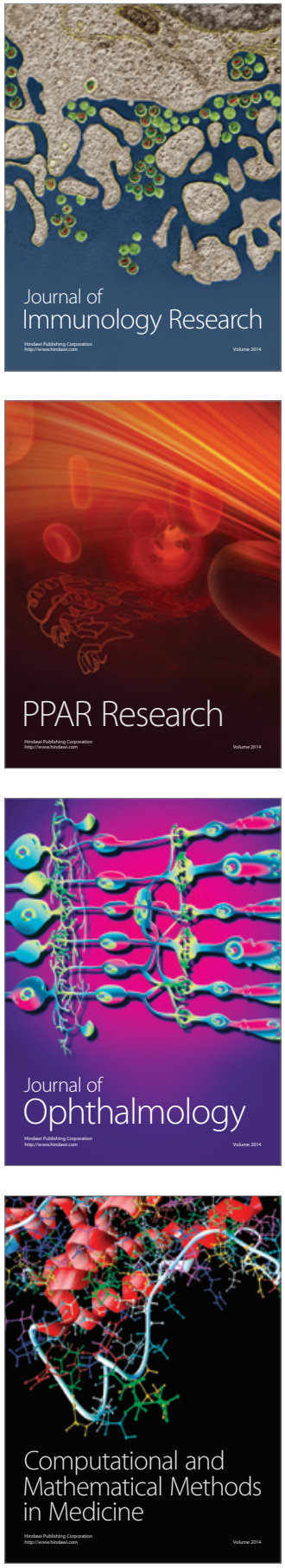

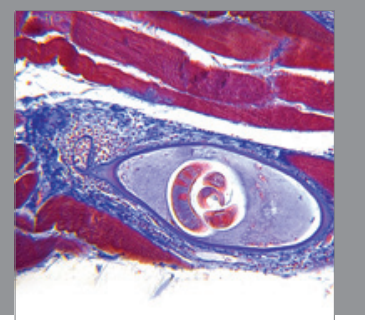

Gastroenterology

Research and Practice
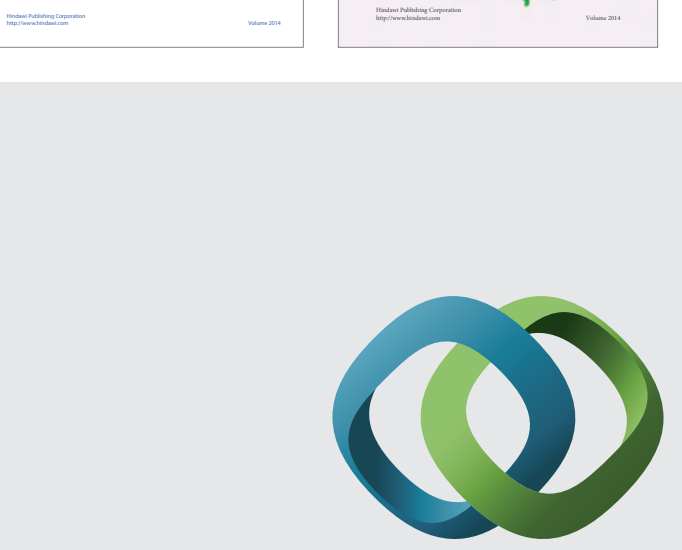

\section{Hindawi}

Submit your manuscripts at

http://www.hindawi.com
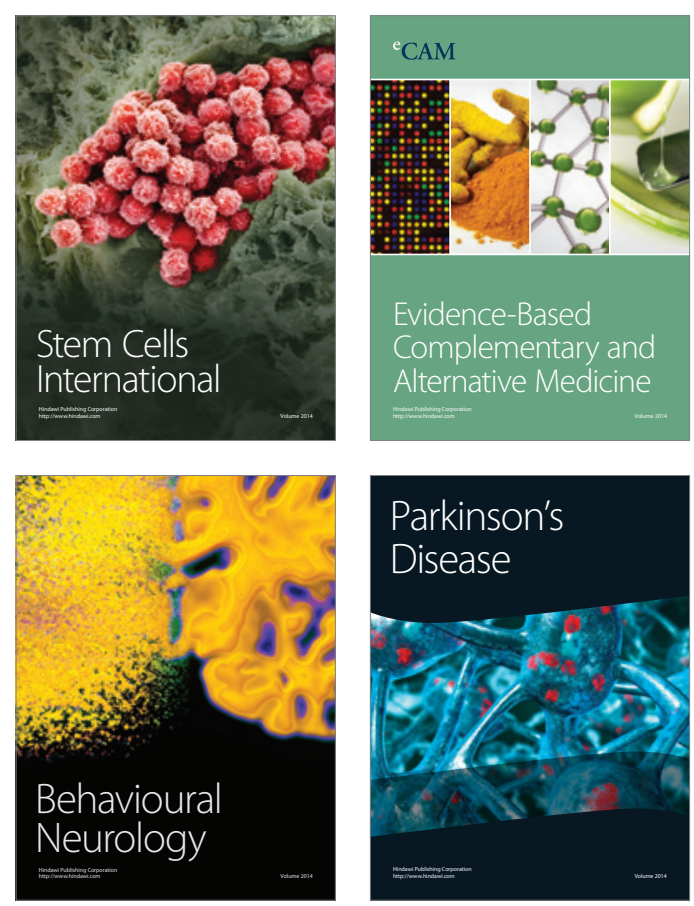

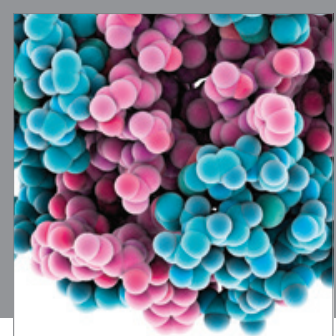

Journal of
Diabetes Research

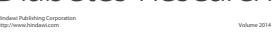

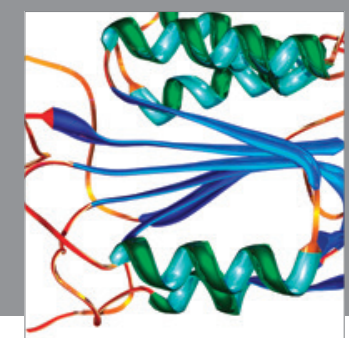

Disease Markers
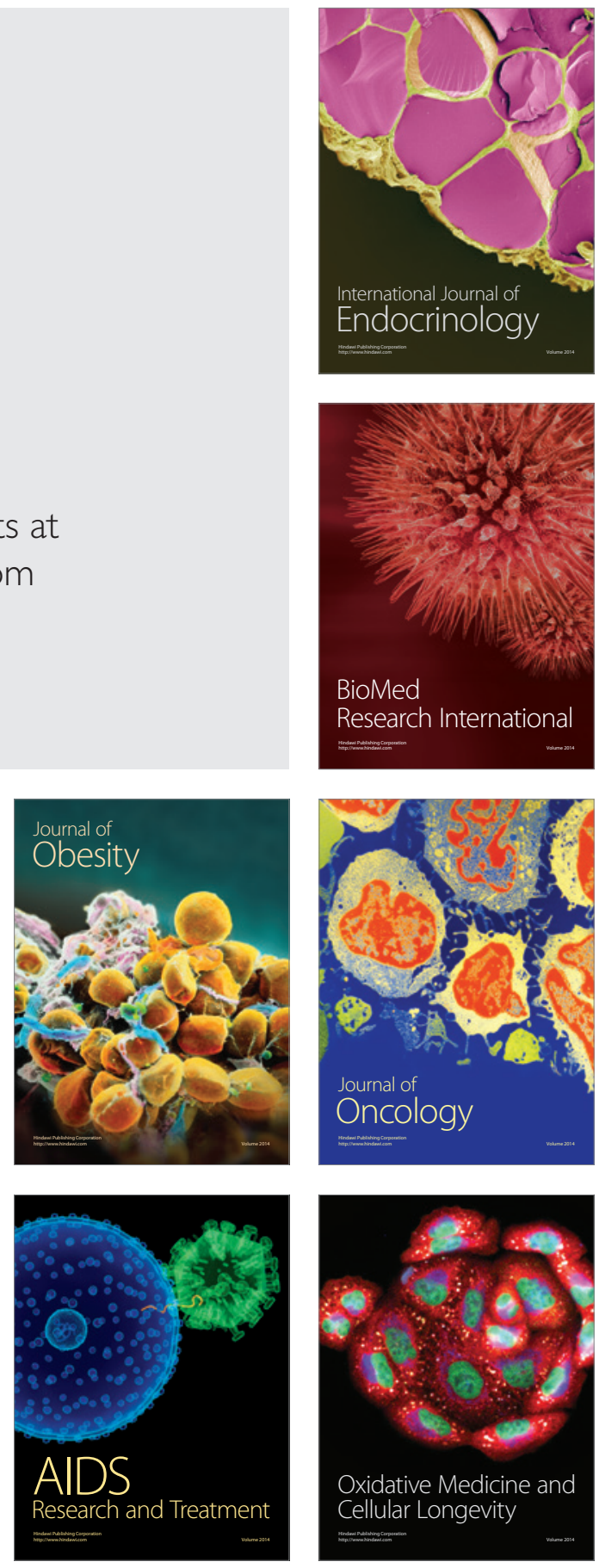